\title{
Al-2 of Aggregatibacter actinomycetemcomitans inhibits Candida albicans biofilm formation
}

\author{
Endang W. Bachtiar ${ }^{1}$, Boy M. Bachtiar ${ }^{1}$, Lucja M. Jarosz ${ }^{2 \dagger}$, Lisa R. Amir ${ }^{1}$, Hari Sunarto ${ }^{3}$, \\ Hadas Ganin ${ }^{4}$, Michael M. Meijler ${ }^{4}$ and Bastiaan P. Krom ${ }^{5 *}$ \\ ${ }^{1}$ Department of Oral Biology, Faculty of Dentistry, Universitas Indonesia, Jakarta, Indonesia \\ 2 Department of Biomedical Engineering, The W.J. Kolff Institute, University Medical Center Groningen and University of Groningen, Groningen, Netherlands \\ ${ }^{3}$ Department of Periodontology, Faculty of Dentistry, Universitas Indonesia, Jakarta, Indonesia \\ ${ }^{4}$ Department of Chemistry, Ben-Gurion University of the Negev, Be'er-Sheva, Israel \\ ${ }^{5}$ Department of Preventive Dentistry, Academic Centre for Dentistry Amsterdam (ACTA), University of Amsterdam and Free University Amsterdam, Amsterdam, \\ Netherlands
}

\author{
Edited by: \\ Alex Mira, Center for Advanced \\ Research in Public Health, Spain \\ Reviewed by: \\ Robert J. C. McLean, TX State \\ University, USA \\ Nick Stephen Jakubovics, \\ Newcastle University, UK \\ *Correspondence: \\ Bastiaan P. Krom, Department of \\ Preventive Dentistry, Academic \\ Centre for Dentistry Amsterdam \\ (ACTA), G. Mahlerlaan 3004, 1081 \\ LA Amsterdam, Netherlands \\ e-mail:b.krom@acta.nl \\ ${ }^{\dagger}$ Present address: \\ Lucja M. Jarosz, Department of Cell \\ Biology, University Medical Center \\ Groningen, Groningen, Netherlands
}

\begin{abstract}
Aggregatibacter actinomycetemcomitans, a Gram-negative bacterium, and Candida albicans, a polymorphic fungus, are both commensals of the oral cavity but both are opportunistic pathogens that can cause oral diseases. A. actinomycetemcomitans produces a quorum-sensing molecule called autoinducer-2 (Al-2), synthesized by LuxS, that plays an important role in expression of virulence factors, in intra- but also in interspecies communication. The aim of this study was to investigate the role of Al-2 based signaling in the interactions between $C$. albicans and $A$. actinomycetemcomitans. A. actinomycetemcomitans adhered to $C$. albicans and inhibited biofilm formation by means of a molecule that was secreted during growth. C. albicans biofilm formation increased significantly when co-cultured with $A$. actinomycetemcomitans luxS, lacking Al-2 production. Addition of wild-type-derived spent medium or synthetic Al-2 to spent medium of the luxS strain, restored inhibition of C. albicans biofilm formation to wild-type levels. Addition of synthetic Al-2 significantly inhibited hypha formation of $C$. albicans possibly explaining the inhibition of biofilm formation. Al-2 of A. actinomycetemcomitans is synthesized by LuxS, accumulates during growth and inhibits $C$. albicans hypha- and biofilm formation. Identifying the molecular mechanisms underlying the interaction between bacteria and fungi may provide important insight into the balance within complex oral microbial communities.
\end{abstract}

Keywords: oral microbiology, interspecies interaction, quorum sensing

\section{INTRODUCTION}

Aggregatibacter actinomycetemcomitans is a non-motile, Gramnegative coccobacillus, which can be found as a commensal in the oral cavity. In addition, it is also the principal cause of aggressive periodontal disease (Saarela et al., 1999). A. actinomycetemcomitans uses chemical signals to sense cell density and alter expression of virulence factors (Novak et al., 2010). This microbial communication is known as quorum sensing (QS) and the only identified cell-cell signaling molecule in A. actinomycetemcomitans this far is autoinducer 2 (AI-2). AI-2, which has been proposed to be a general interspecies, concentration dependent signal, is synthesized by LuxS as a precursor, 4,5,dihydroxy-2,3-pentanedione (DPD), followed by secretion into the medium where it spontaneously undergoes cyclization into AI-2 which accumulates. Interestingly, DPD can thus be converted into several structures that can be recognized by different species (Miller et al., 2004). For instance, Vibrio harveyi produces an unusual furanosyl borate diester ((3aS,6S,6aR)2,2,6,6a-tetrahydroxy-3a-methyltetrahydrofuro[3,2-d][1,3,2] dio xaborol-2-uide), while Salmonella Typhimurium recognizes (2R,4S)-2-methyl-2,3,3,4-tetrahydroxytetrahydrofuran.
In addition, the polymorphic fungus Candida albicans is one of the most commonly isolated fungi from the oral cavity (Cannon and Chaffin, 1999). In healthy individuals, C. albicans grows as a commensal, mostly in the unicellular yeast morphology, but in immune-compromised individuals this species is capable of producing multicellular filamentous forms, a pathogenic morphology (Odds, 1988). The morphological transition from the yeast-to-hyphal mode of growth is influenced by many factors, including $\mathrm{pH}$, nutrient availability, temperature and the presence of QS molecules, that stimulate or repress filamentation as a function of cell density (Hornby et al., 2001; Hazan et al., 2002). Recently, the interaction of bacteria with C. albicans through QS molecules has received increasing attention (Shirtliff et al., 2009). In the oral cavity two Gram-positive bacteria have been shown to affect C. albicans biofilm formation through QS molecules. Streptococcus mutans was shown to inhibit hyphal formation through the QS molecule competence-stimulating peptide (CSP) (Jarosz et al., 2009) and the fatty acid signaling molecule trans2-decenoic acid (Vilchez et al., 2010). In addition, S. gordonii was shown to secrete AI-2, which was shown to repress C. albicans QS by inhibiting the action of farnesol (Bamford et al., 2009) upon 
contact. In the healthy oral cavity a multitude of bacterial species co-exist with C. albicans, both Gram-negative and Gram-positive (Zaura et al., 2009). In contrast to the limited information on the effect of Gram-positive oral bacteria on C. albicans, no information is available on the effect of QS molecules of Gram-negative oral bacteria. Therefore, in the present study, it was our aim to investigate the effect of AI-2 produced by the Gram-negative oral bacterium A. actinomycetemcomitans on C. albicans.

\section{MATERIALS AND METHODS MICROBIAL STRAINS AND GROWTH CONDITIONS}

A. actinomycetemcomitans spp. were routinely cultured for $18 \mathrm{~h}$ at $37^{\circ} \mathrm{C}$ under microaerobic conditions $\left(10 \% \mathrm{CO}_{2}\right)$ on trypticase soy agar or broth containing $0.6 \%$ yeast extract (TSB-YE/Difco). When appropriate, standardized cell suspensions were prepared with a density of $2.1 \times 10^{8} \mathrm{CFU} \mathrm{mL}^{-1}$ as determined with serial dilution plating and determination of the optical density measured at $655 \mathrm{~nm}\left(\mathrm{OD}_{655}\right)$. Escherichia coli was routinely grown in Luria-Bertani broth at $37^{\circ} \mathrm{C}$ with constant aeration. For solid medium, $15 \mathrm{~g}$ agar per liter was added to the liquid medium. When required, ampicillin $\left(100 \mu \mathrm{g} \mathrm{mL}^{-1}\right)$ or kanamycin $(30 \mu \mathrm{g}$ $\mathrm{mL}^{-1}$ ) was added to the medium. C. albicans strain ATCC 10231 or SC5314 were taken from stock cultures frozen in 15\% glycerol at $-80^{\circ} \mathrm{C}$ and sub-cultured twice onto yeast peptone agar plates with $2 \%$ glucose (YPD) or when indicated in yeast nitrogen base medium $\mathrm{pH} 7$, supplemented with $50 \mathrm{mM}$ glucose (YNB).

\section{ADHESION OF A. ACTINOMYCETEMCOMITANS TO C. ALBICANS}

Adhesion of $A$. actinomycetemcomitans Y4 to $C$. albicans strain SC5314 was studied using a Bioflux $1000 Z$ setup. Briefly, C. albicans was seeded into a 48 -well microfluidics plate (Fluxion Biosciences) at an initial optical density measured at $600 \mathrm{~nm}$ of $0.2\left(\mathrm{OD}_{600}=0.2\right)$. After $30 \mathrm{~min}$ adhesion to the bottom plate at $37^{\circ} \mathrm{C}$, flow with prewarmed YNB was started at 0.5 dyne $\mathrm{cm}^{-2}$ for $4 \mathrm{~h}$. A bacterial suspension at an initial $\mathrm{OD}_{600}=0.2$ in phosphate buffered saline (PBS; $10 \mathrm{mM}$ potassium phosphate, $0.15 \mathrm{M}$ sodium chloride, $\mathrm{pH} 7$ ) containing $0.2 \mu \mathrm{L} \mathrm{mL}^{-1}$ Syto9 and $0.2 \mu \mathrm{L} \mathrm{mL}^{-1}$ propidium iodide (Baclight, Invitrogen) was flowed through the microfluidics channels at 0.5 dyne $\mathrm{cm}^{-2}$ and images were captured every $30 \mathrm{~s}$ using the appropriate filter settings. Images were translated to AVI-movies using ImageJ (1.46r).

\section{CONSTRUCTION OF AN A. ACTINOMYCETEMCOMITANS IUxS MUTANT}

A. actinomycetemcomitans (serotype b) was isolated in our periodontal clinic (Universitas Indonesia, Jakarta) from periodontitis patients, with their consent. The isolated bacteria were identified by means of Gram staining, characteristic star-positive colonies on agar plates and tight adherence to surfaces when grown in broth (Slots et al., 1982). Positive clones were confirmed and serotyped using PCR (Suzuki et al., 2001). A single isolate, A. actinomycetemcomitans UI-09, was selected as wildtype strain for all other experiments. In addition, the commonly used A. actinomycetemcomitans Y4 was used as a reference in certain experiments and showed similar results compared to A. actinomycetemcomitans UI-09.

All primers for plasmid construction were designed using A. actinomycetemcomitans database (http://www.oralgen.org, ID for luxS in A. actinomycetemcomitans HK 1651 is AA00516). In order to create a luxS defective mutant, a suicide vector was constructed using the neighbor-joining technique as previously described for Campylobacter jejuni (Bachtiar et al., 2007). Firstly, a 229-bp DNA fragment containing part of the upstream sequence adjacent to $\operatorname{luxS}$ was PCR-amplified using the primer EcoRI-L1 (ACGAATTCAATCCACCGCACTT, forward) and primer BamHI-L2 (TCGGATCCAAGTTTTCTTGTTAGG, reverse). The PCR product was cloned into pBluescript in the forward direction via the EcoRI and BamHI sites, and confirmed by restriction analysis. The construct (pBl-L1) was subsequently introduced into E. coli JM 107. Positive clones were selected on LB agar supplemented with ampicillin, X-Gal and IPTG. Secondly, a 409-bp DNA fragment containing the downstream flanking region of $l u x S$ was amplified using primers BamH1L3 (CATGGATCCGAAGAAGCACATCAA, forward) and XbaI-L4 (ATCTAGAGCAAGTTGCTCGTAA, reverse). The amplified fragment was inserted into pBl-L1 between BamHI and XbaI sites. The resulted intermediate plasmid (pBl-L2) was cut at the unique BamHI site and a 1.4-kbp fragment containing a kanamycin cassette (obtained from vector pMW2 digested with BamHI) was ligated into the plasmid to obtain the suicide plasmid, $\mathrm{pBLkm}{ }^{\mathrm{r}}$.

\section{NATURAL TRANSFORMATION}

A biphasic system for A. actinomycetemcomitans transformation was performed as described previously (Wang et al., 2002). Transformation was done by incubating a suspension of $10^{8} \mathrm{CFU}$ $\mathrm{mL}^{-1}$ of $A$. actinomycetemcomitans at $37^{\circ} \mathrm{C}$ under microaerobic conditions for $3 \mathrm{~h}$. Subsequently, $10 \mu \mathrm{g}$ of the suicide vector was added and cells were incubated for $3 \mathrm{~h}$ at $37^{\circ} \mathrm{C}$. Cells were then harvested and plated on medium supplemented with kanamycin and incubated at $37^{\circ} \mathrm{C}$, under microaerobic conditions for 2 days to select for transformants. Homologous recombination and disruption of $\operatorname{lu} x S$ was confirmed using PCR analysis with primers flanking the target site (EcoRI-L1 and XbaI-L4).

\section{SPENT MEDIUM PREPARATION}

Spent medium of A. actinomycetemcomitans cultures was prepared as described previously (Jarosz et al., 2009). Protein concentration in the spent medium was measured using the Bradford method. Spent medium was diluted in PBS to yield 10 and $100 \mu \mathrm{g}$ $\mathrm{mL}^{-1}$ concentrations and used immediately or stored for short periods of time at $-20^{\circ} \mathrm{C}$. The $\mathrm{pH}$ of the spent medium was adjusted to $\mathrm{pH} 7$.

\section{SYNTHESIS OF (S)-4,5,-DIHYDROXY-2,3-PENTANEDIONE (DPD)}

DPD was synthesized following a procedure published by Ganin et al. (2009). Lyophilized DPD was dissolved in DMSO at $33 \mathrm{mM}$ stock concentrations and stored at $-20^{\circ} \mathrm{C}$ until required. Synthetic DPD concentrations used in the described experiments are assumed to be physiologically relevant as they are in line with concentrations of AI- 2 reported to be produced in saliva-fed natural oral biofilms (Rickard et al., 2008).

\section{BIOFILM FORMATION OF C. ALBICANS AND A. ACTINOMYCETEMCOMITANS}

Quantification of A. actinomycetemcomitans biofilms was achieved by staining with Crystal Violet (CV). 
A. actinomycetemcomitans strains $(5 \mu \mathrm{L})$ were used to inoculated wells of 96-well (flat-bottom) cell culture plates (Sumitomo Bakelite Co., Ltd, Tokyo, Japan) containing $95 \mu \mathrm{L}$ TSB-YE in each well. After, $18 \mathrm{~h}$ of incubation under microaerobic condition, the culture medium containing planktonic cells was removed and the wells were carefully washed with $200 \mu \mathrm{L}$ of distilled water. Adherent bacteria were stained with $50 \mu \mathrm{L}$ of $0.1 \% \mathrm{CV}$ for $15 \mathrm{~min}$ at room temperature. After rinsing twice with $200 \mu \mathrm{L}$ of distilled water, CV bound to the biofilm was extracted with $200 \mu \mathrm{L}$ of $99 \%$ ethanol for $20 \mathrm{~min}$ and quantified by measuring the absorbance at $655 \mathrm{~nm}$ with a microplate reader (Model 3550, Bio-Rad Laboratories, Hercules, CA, USA).

Biofilm formation of $C$. albicans was induced in YNB as previously reported (Krom et al., 2007). Mixed species biofilms of

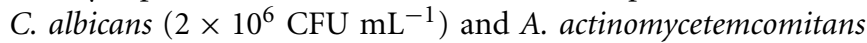
$\left(2.1 \times 10^{7} \mathrm{CFU} \mathrm{mL} \mathrm{m}^{-1}\right)$ were grown in medium containing $70 \%$ YNB and 30\% TSB-YE (vol/vol). Where indicated, the 30\% fresh TSB-YE fraction was replaced by spent medium as described previously (Jarosz et al., 2009). In addition, when indicated, sterile spent medium from the A. actinomycetemcomitans was added to $\mathrm{YNB}$ at 1,10 , and $100 \mu \mathrm{g} \mathrm{mL}^{-1}$ protein concentration. After 24 and $48 \mathrm{~h}$ of growth, biofilm formation on the well of microtiter plates were washed once with PBS and metabolic activity of the biofilms was quantified using [3-(4,5-dimethyl2-thiazolyl)-2,5-diphenyl-2H-tetrazolium bromide] (MTT) as described previously (Krom et al., 2007). Since C. albicans rapidly metabolized $\mathrm{MTT}$, in contrast to A. actinomycetemcomitans (Supplementary Figure S1) this assay could be used to determine $C$. albicans biofilm formation even in co-cultures with A. actinomycetemcomitans. All assays were carried out on at least two separate occasions with at least duplicate determinations on each occasion. Microtiter wells containing only YNB broth but no cells were used as negative controls. To mimic the conditions in the oral cavity initial experiments were also performed in the presence of pooled sterilized human saliva, however no differences were observed relative to experiments without saliva. Therefore, all other experiments were performed without saliva.

\section{INHIBITION OF HYPHA FORMATION}

Hypha formation was assayed as described previously (Jarosz et al., 2009) using C. albicans strain SC5314. The morphology of cells was analyzed using a 20x objective on an inverted microscope (Olympus, Tokyo, Japan).

\section{STATISTICAL ANALYSES}

Differences between means were analyzed for statistical significance using two-tailed Student's $t$-tests. Differences were considered significant when $p \leq 0.05$ level. For multiple comparisons, a One-Way ANOVA followed by a TUKEY HSD test was performed (http://vassarstats.net/anovalu.html).

\section{RESULTS}

\section{ADHESION OF A. ACTINOMYCETEMCOMITANS TO C. ALBICANS}

Adhesion to surfaces is a critical initial step in microbial biofilm formation. Using a microfluidics setup, adhesion under flow conditions were studied. Single A. actinomycetemcomitans Y4 cells adhere to hyphae and yeast cells of $C$. albicans SC5314, as illustrated by the increased green fluorescent spots associated with $C$. albicans (Figure 1). In addition, adhesion of $A$. actinomycetemcomitans to the glass surface could be observed.

\section{EFFECTS OF IUXS DELETION ON BIOFILM FORMATION OF A. ACTINOMYCETEMCOMITANS}

A. actinomycetemcomitans luxS formed significantly less biofilm compared to the wild type strain (Figure 2) but growth rate in planktonic cultures was not affected, in line with previous reports (Novak et al., 2010). Spent medium of a 4 h-old culture of the wild type strain was able to rescue biofilm formation of the luxS strain at $100 \mu \mathrm{g}$ protein $\mathrm{mL}^{-1}$ (Figure 2, left panel). Medium of $6 \mathrm{~h}$-old cultures also rescued the phenotype to a similar, but not medium of 8 and $24 \mathrm{~h}$-old cultures. Addition of synthetic DPD rescued this phenotype specifically at $100 \mathrm{nM}$ DPD, but not at lower or higher concentrations (Figure 2, right panel).
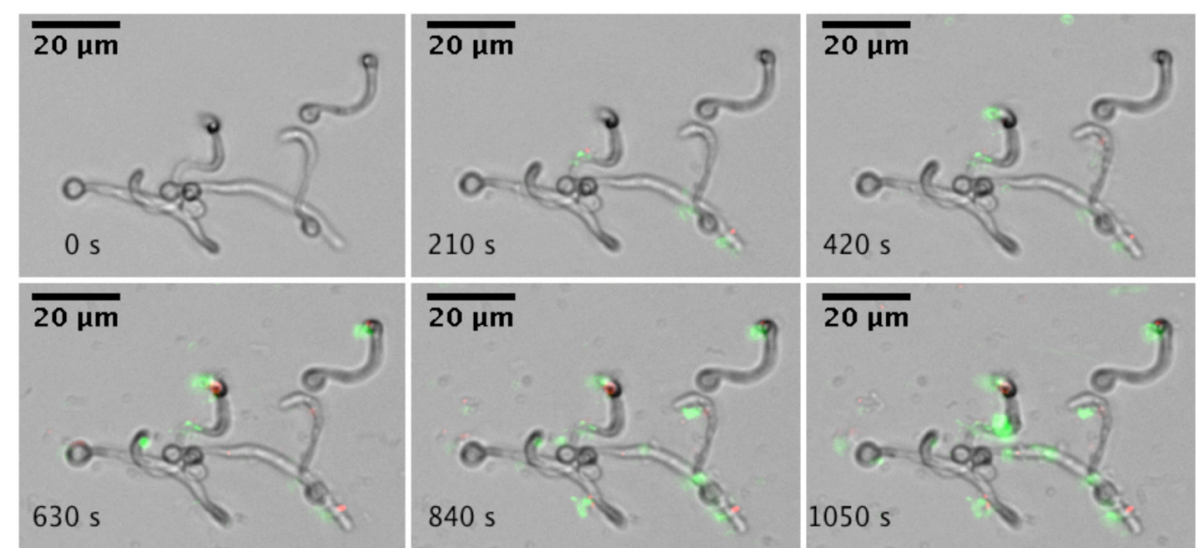

FIGURE 1 | Real-time microscopic analysis of adhesion of A. actinomycetemcomitans to $\boldsymbol{C}$. albicans SC5314. Hyphae of C. albicans SC5314 were allowed to form on the bottom of a microfluidics plate. Bacteria were stained with Syto9 and PI and allowed to adhere to C. albicans SC5314 while flowing at 0.5 dyne $\mathrm{cm}^{-2}$. Images were captured every $30 \mathrm{~s}$ for a total of $10 \mathrm{~min}$ (montage shows images every $210 \mathrm{~s}$ ). Images were edited for brightness and contrast. 


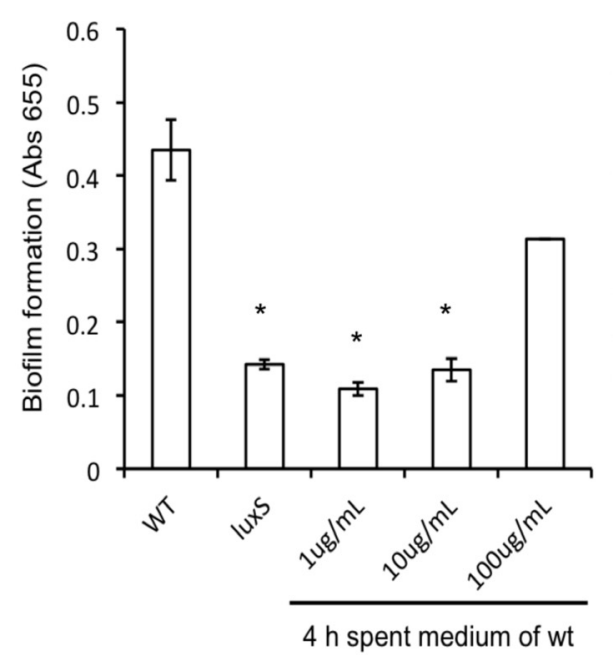

FIGURE 2 |Al-2 plays a role in biofilm formation of $A$. actinomycetemcomitans. Biofilm formation of $A$. actinomycetemcomitans luxS is restored to near wild-type levels by the addition of sterile spent

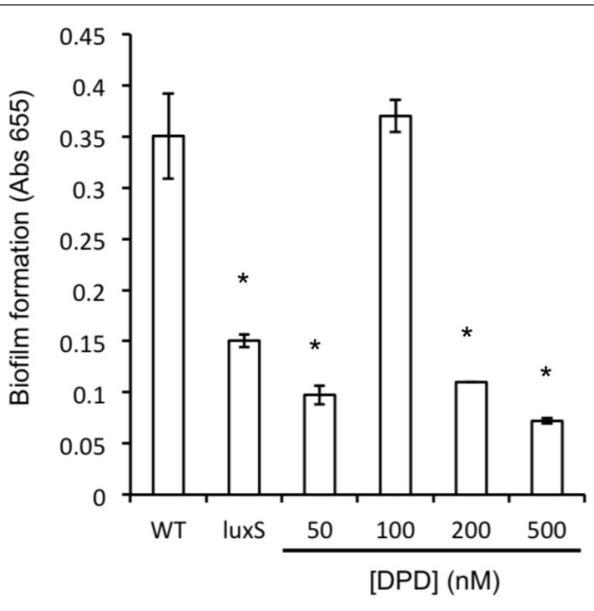

medium (left panel) as well as by the addition of $100 \mathrm{nM}$ synthetic DPD (right panel). *Indicates significantly different from control.

\section{ROLE OF AI-2 ON MIXED SPECIES BIOFILMS OF}

\section{A. ACTINOMYCETEMCOMITANS AND C. ALBICANS}

Compared to mono-species biofilm formation of C. albicans, mixed species biofilms of $C$. albicans with A. actinomycetemcomitans resulted in a reduction of more than $50 \%$ in metabolic activity after $24 \mathrm{~h}$ of culturing (Figure 3A). A similar decrease in biofilm formation was observed after $48 \mathrm{~h}$ of culturing (not shown). Mixed species biofilms of $C$. albicans with A. actinomycetemcomitans luxS had no significant effect compared to mono-species biofilms.

\section{EFFECT OF SECRETED FACTORS ON C. ALBICANS BIOFILM FORMATION}

To further determine whether the inhibition effect was modulated by secreted compounds of the bacteria, the spent medium of the wild type A. actinomycetemcomitans was used as a source of secreted molecules to complement $A$. actinomycetemcomitans luxS. When spent medium from 4 and $6 \mathrm{~h}$-old wild-type cultures was added, the A. actinomycetemcomitans luxS mutant was able to inhibit $C$. albicans biofilm formation, but this inhibition was not observed for spent medium derived from 8 and 24-h old cultures (Figure 3B). Conversely, when spent medium of $A$. actinomycetemcomitans $l u x S$ was added to mixed species biofilms of $C$. albicans and $A$. actinomycetemcomitans luxS, no significant inhibitions of biofilm formation was observed (Supplementary Figure S2).

\section{EFFECT OF SYNTHETIC DPD ON C. ALBICANS BIOFILM GROWTH AND HYPHA FORMATION}

When synthetic DPD was added to spent medium of A. actinomycetemcomitans luxS during $C$. albicans biofilm growth, a concentration dependent inhibition of $C$. albicans biofilm formation was observed with the maximum inhibition reached at $100 \mathrm{nM}$ DPD (Figure 4, right panel). Hypha formation is a key process in biofilm formation. Synthetic DPD was added to C. albicans under hypha inducing conditions. DPD inhibited hypha formation by 30 and $70 \%$ at $100 \mathrm{nM}$ and $1 \mu \mathrm{M}$, respectively (Figure 4, left panel).

\section{A. ACTINOMYCETEMCOMITANS SPENT MEDIUM DISRUPTS ESTABLISHED C. ALBICANS BIOFILM}

To test the ability of A. actinomycetemcomitans to disrupt C. albicans biofilms, we challenged established biofilms ( $24 \mathrm{~h}$ old) of C. albicans with spent medium from A. actinomycetemcomitans WT and $l u x S$ cultures of increasing age. A culture age dependent decrease in viability was observed when $C$. albicans biofilms were exposed to spent medium of cultures of the WT strain, but not to spent medium derived from the same aged cultures of the luxS strain (Figure 5).

\section{DISCUSSION}

A. actinomycetemcomitans is related to severe periodontitis. In addition, several studies have reported on the isolation of a large number Candida spp., from periodontal pockets of patients with periodontitis (Jarvensivu et al., 2004; Urzua et al., 2008). Recently, co-isolation of Candida spp. and A. actinomycetemcomitans was correlated with the occurrence of severe periodontitis (Brusca et al., 2010). However, due to limited information it is currently unclear if $C$. albicans participates in the etiology of any kind of periodontitis. Biofilms are the most common mode of growth of Candida spp., as observed in vivo. Therefore, interspecies interactions occur preferentially in mixed species biofilms such as those found in the periodontal pocket. A better fundamental understanding of interspecies interaction in the oral cavity is therefore of great relevance. Here we show for the first time that a commonly isolated periodontal pathogen A. actinomycetemcomitans adheres to $C$. albicans under flow condition (Figure 1). Physical interactions, such as adhesion are initial and probably critical stages in microbial biofilm formation. Following adhesion growth 

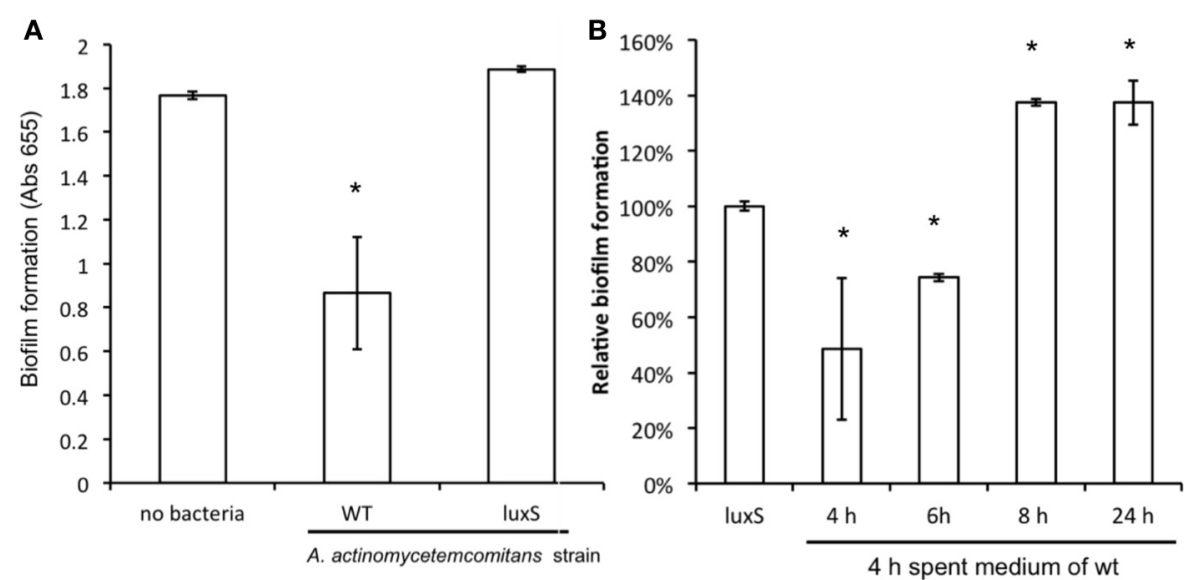

FIGURE 3 | The effect of co-culture with A. actinomycetemcomitans on C. albicans ATCC 10231 biofilm formation. (A) C. albicans biofilms were grown without any bacteria or with $A$. actinomycetemcomitans wild-type or luxS mutant. Biofilm formation, quantified using the MTT assay, was measured after $24 \mathrm{~h}$ of growth. Data represent the mean and standard deviations of six biofilms grown on two separate occasions. (B) The effect of spent medium on co-cultures between $C$. albicans and A. actinomycetemcomitans luxS. Spent medium of
A. actinomycetemcomitans wild-type cultures grown for different times was added to the mixed species culture of $C$. albicans and

A. actinomycetemcomitans luxS. Biofilm formation was quantified after $24 \mathrm{~h}$ of growth using the MTT assay. The relative biofilm formation compared to C. albicans $+A$. actinomycetemcomitans luxS without any spent medium was calculated. Data represent the mean and standard deviations of six biofilms grown on two separate occasions. *Indicates significantly different from control.

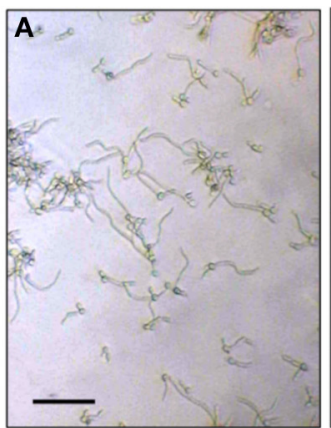

D

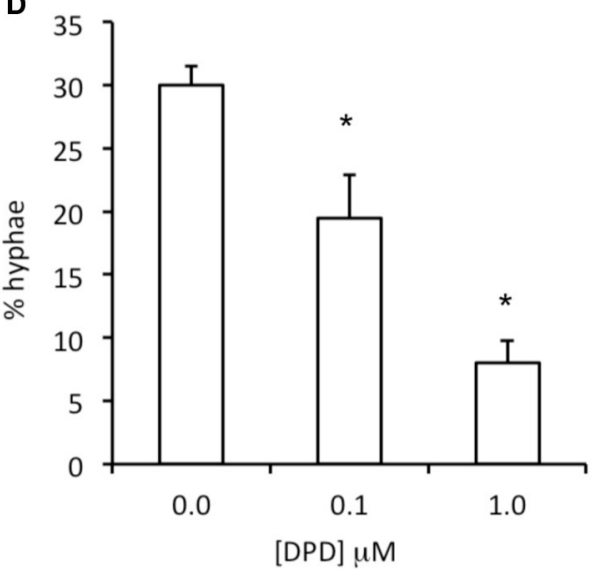

FIGURE 4 | Effect of synthetic DPD on C. albicans SC5134.

Hypha-formation was induced by switching fresh cultures to $37^{\circ} \mathrm{C}$ for 3-4 h. Representative microscopic images show a decreased hypha formation $(\mathbf{A}=$ control, $\mathbf{B}=0.1 \mu \mathrm{M}$ DPD, $\mathbf{C}=1.0 \mu \mathrm{M}$ DPD; bar represent $40 \mu \mathrm{m}$ for all images). Hyphae and yeast morphologies were
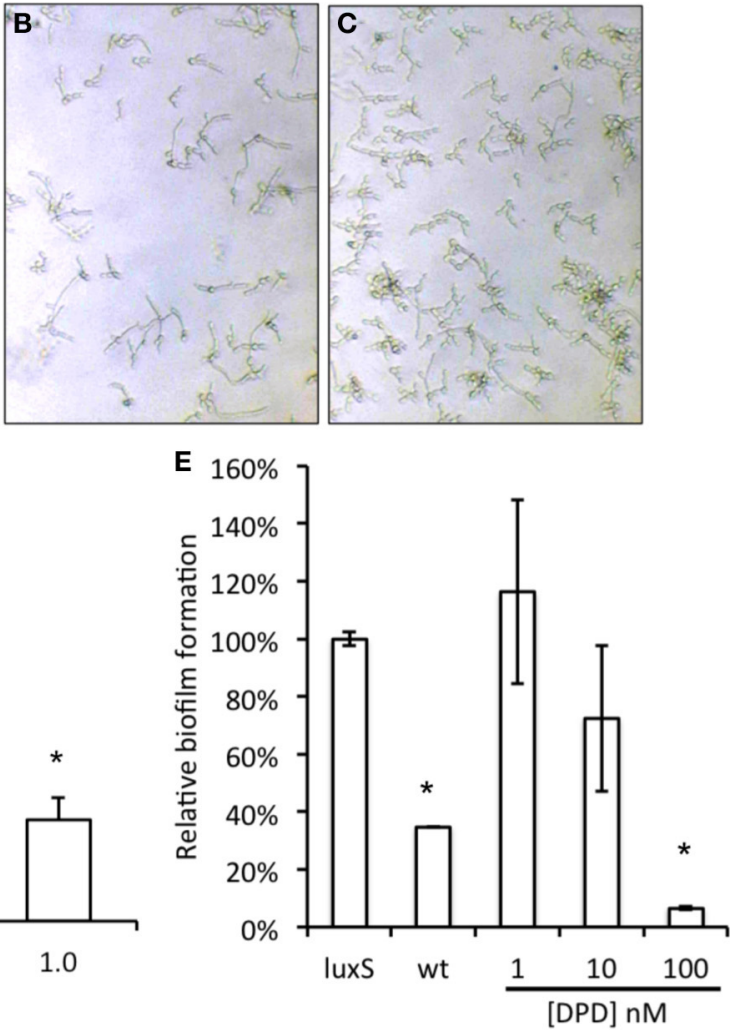

counted and plotted as \% of all cells (D). The results represent the mean of two independent experiments, each consisting of at least 100 cells per sample. Synthetic DPD added to spent medium of A. actinomycetemcomitans luxS inhibits C. albicans SC5314 biofilm formation (E). *Indicates significantly different from control. 


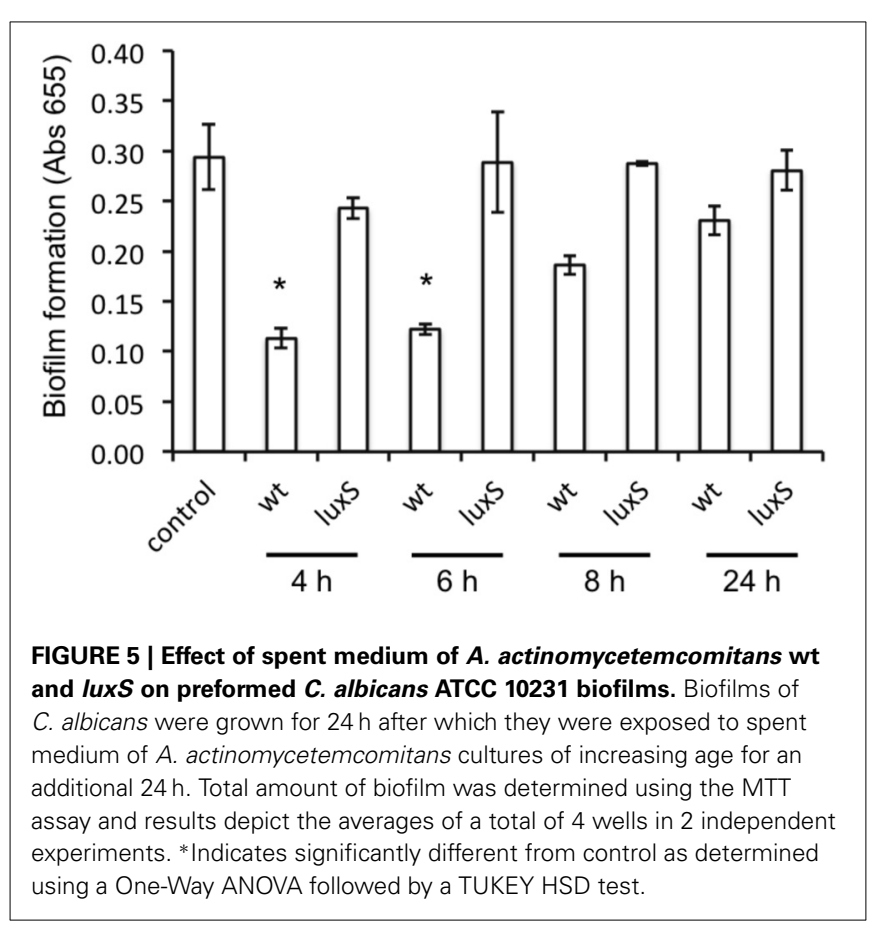

and accumulation results in interspecies chemical interactions, amongst others mediated by quorum sensing (Jarosz and Krom, 2011).

AI-2 activity has been discovered in spent culture supernatants of many oral bacteria (Fong et al., 2001; Blehert et al., 2003; Wen and Burne, 2004). However, the function of AI-2 as a general bacterial signaling molecule is an issue that is yet to be resolved. Several studies have suggested that AI-2 is involved in biofilm formation (Chung et al., 2001; Yoshida et al., 2005). On the other hand, it has also been suggested that the main function of the LuxS enzyme is in the regulation of metabolic processes (Winzer et al., 2002). Inactivation of luxS in A. actinomycetemcomitans does not affect growth rates but does result in phenotypic alterations, including biofilm formation and reduced colonization in various experimental infection models (Hardie and Heurlier, 2008). Our data demonstrated that biofilm formation of the A. actinomycetemcomitans luxS strain was decreased as compared with its parent strain. Complementation of the luxS deletion by both spent medium of wild-type A. actinomycetemcomitans as well as by addition of synthetic DPD would indicate that the luxS mutant phenotype is due to absence of the quorum sensing molecule and not related to any metabolic defect. It should however be noted that it is not straightforward to uncouple signaling and metabolic functions of LuxS (Redanz et al., 2012). The remarkable concentration dependent complementation of the luxS mutant by DPD—reaching a maximum at $100 \mathrm{nM}$, followed by a sharp decrease at higher concentrations is interesting and currently we are unable to explain this specific behavior. It is however important to notice that such a behavior has been observed in another study. Rickard and coworkers described a similar sharp concentration optimum for synthetic AI-2 in a mixed-species biofilm model consisting of Actinomyces naeslundii and Streptococcus oralis be it at approximately 100-fold lower concentration (Rickard et al., 2006). Spent medium of $8 \mathrm{~h}$ cultures and older no longer inhibited C. albicans biofilm formation (see Figure 5). This could be related to decreased AI-2 presence at later stages of growth which is in line with the growthphase specific production of AI-2, higher in the first $6 \mathrm{~h}$ of growth compared to later stages, observed in several oral species (Fong et al., 2001; Blehert et al., 2003; Wen and Burne, 2004).

Bamford et al. showed that $S$. gordonii increases C. albicans biofilm formation in a LuxS dependent fashion, however, no effect of synthetic DPD on C. albicans hypha formation was observed (Bamford et al., 2009). In contrast, we observed that the exogenous addition of AI-2/DPD to the growth medium restored the luxS phenotype in a dose-dependent manner and that addition of synthetic DPD inhibited hypha formation of C. albicans. This effect was only seen in medium consisting of $70 \% \mathrm{YNB} / 30 \%$ $\mathrm{TSB}$, and not in YNB alone. In contrast to Bamford we did not use saliva in the germ-tube assay. Both minimal medium and saliva are strong inducers of hypha formation and this might explain the observed different response of C. albicans to synthetic DPD. Additionally, use of different $C$. albicans strains in the present study compared to Bamford and coworkers could account for the observed differences in response. Alternatively, the different response under different hypha inducing conditions could indicate a difference in the chemical structure of AI-2 produced by S. gordonii or A. actinomycetemcomitans, and intriguingly that C. albicans is able to recognize this chemical difference. Detailed chemical characterization of the AI-2 for both species should be performed to further support this statement.

Communication within oral microorganisms involves several classes of signal molecules; autoinducing peptides synthesized by Gram-positive bacteria, such as competence-stimulating peptide of S. mutans (Jarosz et al., 2009), fatty acid signaling molecules such as farnesol and trans-2-decenoic acid (SDSF) (Vilchez et al., 2010) and AI-2 as a proposed universal signal produced by both Gram-positive and Gram-negative bacteria (Federle and Bassler, 2003). The different responses of $C$. albicans to AI-2, depending on its origin, is intriguing and could illustrate that the already complicated interspecies chemical communication might be even more complicated, but a more extensive study is needed to provide solid evidence for such a tempting, but speculative conclusion.

Oral biofilms are very complex multi-species communities in which we assume that interspecies interaction plays a role in establishing and maintaining a balance. A recent study on bacterial and yeast colonization in a group of mucositis patients showed a complete absence of A. actinomycetemcomitans and a more than average presence of $C$. albicans (Laheij et al., 2012). As hypha formation is a pivotal step in C. albicans biofilm formation (Krueger et al., 2004) and adhesion, the LuxS mediated interaction with A. actinomycetemcomitans would decrease fungal biofilm formation. Additionally, the function of LuxS is related to the regulation of bacteria virulence factors, including cytolethal distending toxin (CDT), as reported in Campylobacter jejuni (Jeon et al., 2005). For A. actinomycetemcomitans it has been shown that CDT (CDTB) is toxic to the yeast Saccharomyces cereviseae (Matangkasombut et al., 2010). There are therefore potentially multiple systems, one based on 
a secreted QS molecule and a second on a toxin, involved in the observed negative interactions between A. actinomycetemcomitans and $C$. albicans. A preliminary study indicated that spent medium of wild-type $A$. actinomycetemcomitans has no toxic effects on C. albicans (Supplementary Figure S3). Similar dual mechanisms of inhibition have been observed between Staphylococcus aureus and Pseudomonas aeruginosa (Li and Krom, unpublished data). It is tempting to hypothesize that the QS molecule is sensed as a defense mechanism to protect against the upcoming battle with a toxin-producing competitor (Jarosz et al., 2011). Additional studies are required to further elucidate the molecular and biochemical mechanisms involved in the interspecies interaction between A. actinomycetemcomitans and C. albicans.

\section{ACKNOWLEDGMENTS}

We thank Maysyaroh S. Si and Dessy Sulistya Ashari S. Si for technical assistance. This research was supported in part by a grant from Universitas Indonesia (RUUI), Indonesia, to Boy M. Bachtiar, and by the Division for Earth and Life Sciences (ALW) with financial aid from the Netherlands Organization for Scientific Research (NWO). Michael M. Meijler and Bastiaan P. Krom were supported by a Young Investigator Grant of the Human Frontier Science Program (HFSP; RGY0072/2007). Bastiaan P. Krom is supported by a grant from the University of Amsterdam for research into the focal point "Oral Infections and Inflammation.”

\section{SUPPLEMENTARY MATERIAL}

The Supplementary Material for this article can be found online at: http://www.frontiersin.org/journal/10.3389/fcimb.2014. 00094/abstract

Supplementary Figure S1 | Comparison of MTT and XTT reduction by C. albicans SC5314 and A. actinomycetemcomitans. Metabolic activity of C. albicans yields a purple or orange color upon reduction of MTT and XTT respectively. Incubation of $A$. actinomycetemcomitans does not result in a significant color change.

\section{Supplementary Figure S2 | Complementation of the luxS-effect using spent medium of wild-type $\boldsymbol{A}$. actinomycetemcomitans. Addition of 4 and 6 h-old wt-spent medium to mixed species biofilms of $C$. albicans ATCC 10231 and $A$. actinomycetemcomitans results in inhibition of biofilm formation. Addition of spent medium of $A$. actinomycetemcomitans luxS did not affect biofilm formation.}

\section{Supplementary Figure S3 | Effect of spent medium of wild-type A. actinomycetemcomitans on growth kinetics of $C$. albicans SC5134. An overnight culture of $C$. albicans was diluted 1:20 or 1:50 in medium consisting of $70 \%$ YNB $+30 \%$ filter sterilized 4 h-old spent medium of wild-type $A$. actinomycetemcomitans. As a control, the same inoculum was grown in $70 \%$ YNB $+30 \%$ fresh TSB + YE. No significant differences were observed illustrating the absence of any toxicity related agent in the spent medium of wild-type $A$. actinomycetemcomitans.}

\section{REFERENCES}

Bachtiar, B. M., Coloe, P. J., and Fry, B. N. (2007). Knockout mutagenesis of the $k p s E$ gene of Campylobacter jejuni 81116 and its involvement in bacterium-host interactions. FEMS Immunol. Med. Microbiol. 49, 149-154. doi: 10.1111/j.1574695X.2006.00182.x
Bamford, C. V., D’Mello, A., and Nobbs, A. H. (2009). Streptococcus gordonii modulates Candida albicans biofilm formation through intergeneric communication. Infect. Immun. 77, 3696-3704. doi: 10.1128/IAI.00438-09

Blehert, D. S., Palmer, R. J. Jr., Xavier, J. B., Almeida, J. S., and Kolenbrander, P. E. (2003). Autoinducer 2 production by Streptococcus gordonii DL1 and the biofilm phenotype of a luxS mutant are influenced by nutritional conditions. J. Bacteriol. 185, 4851-4860. doi: 10.1128/JB.185.16.4851-4860.2003

Brusca, M. I., Rosa, A., Albaina, O., Moragues, M. D., Verdugo, F., and Ponton, J. (2010). The impact of oral contraceptives on women's periodontal health and the subgingival occurrence of aggressive periodontopathogens and Candida species. J. Periodontol. 81, 1010-1018. doi: 10.1902/jop.2010.090575

Cannon, R. D., and Chaffin, W. L. (1999). Oral colonization by Candida albicans. Crit. Rev. Oral. Biol. Med. 10, 359-383. doi: 10.1177/1045441199010 0030701

Chung, W. O., Park, Y., Lamont, R. J., McNab, R., Barbieri, B., and Demuth, D. R. (2001). Signaling system in Porphyromonas gingivalis based on a LuxS protein. J. Bacteriol. 183, 3903-3909. doi: 10.1128/JB.183.13.3903-3909.2001

Federle, M. J., and Bassler, B. L. (2003). Interspecies communication in bacteria. J. Clin. Invest. 112, 1291-1299. doi: 10.1172/JCI20195

Fong, K. P., Chung, W. O., Lamont, R. J., and Demuth, D. R. (2001). Intra- and interspecies regulation of gene expression by Actinobacillus actinomycetemcomitans LuxS. Infect. Immun. 69, 7625-7634. doi: 10.1128/IAI.69.12.76257634.2001

Ganin, H., Tang, X., and Meijler, M. M. (2009). Inhibition of Pseudomonas aeruginosa quorum sensing by AI-2 analogs. Bioorg. Med. Chem. Lett. 19, 3941-3944. doi: 10.1016/j.bmcl.2009.03.163

Hardie, K. R., and Heurlier, K. (2008). Establishing bacterial communities by "word of mouth": LuxS and autoinducer 2 in biofilm development. Nat. Rev. Microbiol. 6, 635-643. doi: 10.1038/nrmicro1916

Hazan, I., Sepulveda-Becerra, M., and Liu, H. (2002). Hyphal elongation is regulated independently of cell cycle in Candida albicans. Mol. Biol. Cell. 13, 134-145. doi: 10.1091/mbc.01-03-0116

Hornby, J. M., Jensen, E. C., Lisec, A. D., Tasto, J. J., Jahnke, B., Shoemaker, R., et al. (2001). Quorum sensing in the dimorphic fungus Candida albicans is mediated by farnesol. Appl. Environ. Microbiol. 67, 2982-2992. doi: 10.1128/AEM.67.7.2982-2992.2001

Jarosz, L. M., Deng, D. M., van der Mei, H. C., Crielaard, W., and Krom, B. P. (2009). Streptococcus mutans competence-stimulating peptide inhibits Candida albicans hypha formation. Eukaryot. Cell 8, 1658-1664. doi: 10.1128/EC.00070-09

Jarosz, L. M., and Krom, B. P. (2011). Rapid screening method for compounds that affect the growth and germination of Candida albicans, using a real-time PCR thermocycler. Appl. Environ. Microbiol. 77, 8193-8196. doi: 10.1128/AEM.06227-11

Jarosz, L. M., Ovchinnikova, E. S., Meijler, M. M., and Krom, B. P. (2011). Microbial spy games and host response: roles of a Pseudomonas aeruginosa small molecule in communication with other species. PLoS Pathogens 7:e1002312. doi: 10.1371/journal.ppat.1002312

Jarvensivu, A., Hietanen, J., Rautemaa, R., Sorsa, T., and Richardson, M. (2004). Candida yeasts in chronic periodontitis tissues and subgingival microbial biofilms in vivo. Oral Dis. 10, 106-112. doi: 10.1046/j.1354-523X.2003.00978.x

Jeon, B., Itoh, K., and Ryu, S. (2005). Promoter analysis of cytolethal distending toxin genes (cdtA, B, and C) and effect of a luxS mutation on CDT production in Campylobacter jejuni. Microbiol. Immunol. 49, 599-603. doi: 10.1111/j.13480421.2005.tb03651.x

Krom, B. P., Cohen, J. B., McElhaney Feser, G. E., and Cihlar, R. L. (2007). Optimized candidal biofilm microtiter assay. J. Microbiol. Methods 68, 421-423. doi: 10.1016/j.mimet.2006.08.003

Krueger, K. E., Ghosh, A. K., Krom, B. P., and Cihlar, R. L. (2004). Deletion of the NOT4 gene impairs hyphal development and pathogenicity in Candida albicans. Microbiology 150, 229-240. doi: 10.1099/mic.0.26792-0

Laheij, A. M., de Soet, J. J., von dem Borne, P. A., Kuijper, E. J., Kraneveld, E. A., van Loveren, C., et al. (2012). Oral bacteria and yeasts in relationship to oral ulcerations in hematopoietic stem cell transplant recipients. Support. Care Cancer 20, 3231-3240. doi: 10.1007/s00520-012-1463-2

Matangkasombut, O., Wattanawaraporn, R., Tsuruda, K., Ohara, M., Sugai, M., and Mongkolsuk, S. (2010). Cytolethal distending toxin from Aggregatibacter actinomycetemcomitans induces DNA damage, S/G2 cell cycle arrest, and caspaseindependent death in a Saccharomyces cerevisiae model. Infect. Immun. 78, 783-792. doi: 10.1128/IAI.00857-09 
Miller, S. T., Xavier, K. B., Campagna, S. R., Taga, M. E., Semmelhack, M. F., Bassler, B. L., et al. (2004). Salmonella typhimurium recognizes a chemically distinct form of the bacterial quorum-sensing signal AI-2. Mol. Cell 15, 677-687. doi: 10.1016/j.molcel.2004.07.020

Novak, E. A., Shao, H., Daep, C. A., and Demuth, D. R. (2010). Autoinducer-2 and QseC control biofilm formation and in vivo virulence of Aggregatibacter actinomycetemcomitans. Infect. Immun. 78, 2919-2926. doi: 10.1128/IAI. 01376-09

Odds, F. C. (1988). Candida and Candidosis. London: Bailliere Tindall.

Redanz, S., Standar, K., Podbielski, A., and Kreikemeyer, B. (2012). Heterologous expression of sahH reveals that biofilm formation is autoinducer-2-independent in Streptococcus sanguinis but is associated with an intact activated methionine cycle. J. Biol. Chem. 287, 36111-36122. doi: 10.1074/jbc.M112.379230

Rickard, A. H., Campagna, S. R., and Kolenbrander, P. E. (2008). Autoinducer-2 is produced in saliva-fed flow conditions relevant to natural oral biofilms. J. Appl. Microbiol. 105, 2096-2103. doi: 10.1111/j.1365-2672.2008.03910.x

Rickard, A. H., Palmer, R. J. Jr., Blehert, D. S., Campagna, S. R., Semmelhack, M. F., Egland, P. G., et al. (2006). Autoinducer 2: a concentration-dependent signal for mutualistic bacterial biofilm growth. Mol. Microbiol. 60, 1446-1456. doi: 10.1111/j.1365-2958.2006.05202.x

Saarela, M. H., Dogan, B., Alaluusua, S., and Asikainen, S. (1999). Persistence of oral colonization by the same Actinobacillus actinomycetemcomitans strain(s). J. Periodontol. 70, 504-509. doi: 10.1902/jop.1999.70.5.504

Shirtliff, M. E., Peters, B. M., and Jabra-Rizk, M. A. (2009). Cross-kingdom interactions: Candida albicans and bacteria. FEMS Microbiol. Lett. 299, 1-8. doi: 10.1111/j.1574-6968.2009.01668.x

Slots, J., Zambon, J. J., Rosling, B. G., Reynolds, H. S., Christersson, L. A., and Genco, R. J. (1982). Actinobacillus actinomycetemcomitans in human periodontal disease. Association, serology, leukotoxicity, and treatment. J. Periodontal Res. 17, 447-448. doi: 10.1111/j.1600-0765.1982.tb02022.x

Suzuki, N., Nakano, Y., Yoshida, Y., Ikeda, D., and Koga, T. (2001). Identification of Actinobacillus actinomycetemcomitans serotypes by multiplex PCR. J. Clin. Microbiol. 39, 2002-2005. doi: 10.1128/JCM.39.5.2002-2005.2001

Urzua, B., Hermosilla, G., Gamonal, J., Morales-Bozo, I., Canals, M., Barahona, S., et al. (2008). Yeast diversity in the oral microbiota of subjects with periodontitis: Candida albicans and Candida dubliniensis colonize the periodontal pockets. Med. Mycol. 46, 783-793. doi: 10.1080/13693780802060899

Vilchez, R., Lemme, A., Ballhausen, B., Thiel, V., Schulz, S., Jansen, R., et al. (2010). Streptococcus mutans inhibits Candida albicans hyphal formation by the fatty acid signaling molecule trans-2-decenoic acid (SDSF). Chembiochem 11, 1552-1562. doi: 10.1002/cbic.201000086

Wang, Y., Goodman, S. D., Redfield, R. J., and Chen, C. (2002). Natural transformation and DNA uptake signal sequences in Actinobacillus actinomycetemcomitans. J. Bacteriol. 184, 3442-3449. doi: 10.1128/JB.184.13.34423449.2002

Wen, Z. T., and Burne, R. A. (2004). LuxS-mediated signaling in Streptococcus mutans is involved in regulation of acid and oxidative stress tolerance and biofilm formation. J. Bacteriol. 186, 2682-2691. doi: 10.1128/JB.186.9.26822691.2004

Winzer, K., Hardie, K. R., Burgess, N., Doherty, N., Kirke, D., Holden, M. T., et al. (2002). LuxS: its role in central metabolism and the in vitro synthesis of 4-hydroxy-5-methyl-3(2H)-furanone. Microbiology 148, 909-922.

Yoshida, A., Ansai, T., Takehara, T., and Kuramitsu, H. K. (2005). LuxSbased signaling affects Streptococcus mutans biofilm formation. Appl. Environ. Microbiol. 71, 2372-2380. doi: 10.1128/AEM.71.5.23722380.2005

Zaura, E., Keijser, B. J., Huse, S. M., and Crielaard, W. (2009). Defining the healthy "core microbiome" of oral microbial communities. BMC Microbiol. 9:259. doi: $10.1186 / 1471-2180-9-259$

Conflict of Interest Statement: The authors declare that the research was conducted in the absence of any commercial or financial relationships that could be construed as a potential conflict of interest.

Received: 27 March 2014; accepted: 26 June 2014; published online: 21 July 2014.

Citation: Bachtiar EW, Bachtiar BM, Jarosz LM, Amir LR, Sunarto H, Ganin H, Meijler MM and Krom BP (2014) AI-2 of Aggregatibacter actinomycetemcomitans inhibits Candida albicans biofilm formation. Front. Cell. Infect. Microbiol. 4:94. doi: 10.3389/fcimb.2014.00094

This article was submitted to the journal Frontiers in Cellular and Infection Microbiology.

Copyright (c) 2014 Bachtiar, Bachtiar, Jarosz, Amir, Sunarto, Ganin, Meijler and Krom. This is an open-access article distributed under the terms of the Creative Commons Attribution License (CC BY). The use, distribution or reproduction in other forums is permitted, provided the original author(s) or licensor are credited and that the original publication in this journal is cited, in accordance with accepted academic practice. No use, distribution or reproduction is permitted which does not comply with these terms. 